\title{
Probabilistic analysis for osseointegration process of hollow stem used in un-cemented hip prosthesis
}

\section{Analyse probabiliste pour le processus d'ostéo-intégration de tige trouée utilisée dans la prothèse de la hanche non cimentée}

\author{
Ghias Kharmanda ${ }^{1,2}$, Abdallah Shokry ${ }^{3}$, Imad Antypas ${ }^{4}$, Abdelkhalak El-Hami ${ }^{2}$ \\ ${ }^{1}$ Department of Biomedical Engineering, Lund University, Sweden, Ghias.Kharmanda@bme.lth.se \\ ${ }^{2}$ Mechanics Laboratory of Normandy, INSA Rouen, St Etienne du Rouvray, France, Abdelkhalak.elhami@insa-rouen.fr \\ ${ }^{3}$ Department of Mechanical Engineering, Fayoum University, Fayoum, Egypt, abdallah.shokry@fayoum.edu.eg \\ ${ }^{4}$ State Technical University, Rostov-on-Don, Russian Federation, imad.antypas@mail.ru
}

\begin{abstract}
This paper describes the application of optimization and probabilistic methods to a hollow stem design implanted in a proximal femur. The objective is to introduce a hollow stem as a robust tool for fixation. Introducing the hollow stem and taking advantage of shape optimization with objectives in stresses, the regions where stresses shielding exists can be reduced as a conclusion. Probabilistic methods allow variations in factors which control the hole fixation of the implanted femur (the input parameters) to be taken into account in determining its performance. The probabilistic analysis is applied on the resulting hollow stem to simulate the variation factors of the bone ingrowth into holes. The fixation values are generated randomly using Monte Carlo simulation (MCS). Monte Carlo sampling techniques were applied and the maximum stress in the cancellous bone was chosen as a performance indicator. A simple 2D implantbone study of solid and hollow stem designs is carried out with a high level of confidence $99.9 \%$ taking into account statistical uncertainties.

RÉSUMÉ. Cet article décrit l'application d'optimisation et méthodes probabilistes à la conception d'une tige trouée implantée dans un fémur proximal. L'objectif est d'introduire une tige trouée comme un outil robuste pour la fixation. Introduire la tige trouée et profitant de l'optimisation de forme avec des objectifs de contraintes, les régions où il existe des contraintes de blindage peuvent être réduites. Méthodes probabilistes permettent des variations dans les facteurs qui contrôlent la fixation de trou du fémur implanté (paramètres d'entrée) à prendre en considération pour déterminer sa performance. L'analyse probabiliste est appliquée sur la tige trouée résultante pour simuler les facteurs de variation de la croissance osseuse dans les trous. Les valeurs de fixation sont générées aléatoirement à l'aide de simulation de Monte Carlo (MC). Techniques d'échantillonnage de Monte Carlo ont été appliquées et la contrainte maximale dans l'os spongieux a été choisie comme un indicateur de performance. Une étude simple 2D implant d'os de conceptions de tiges pleines et trouées est réalisée avec un haut niveau de confiance de 99,9\% en tenant compte des incertitudes statistiques.
\end{abstract}

KEYWORDS. Hip Prosthesis, Probabilistic Analysis, Finite Element Analysis, Monte Carlo simulation.

MOTS-CLÉS. Prothèse de hanche, simulation de Monte Carlo, analyse probabiliste, analyse par éléments finis.

\section{Introduction}

There are several factors that need to be considered when analyzing the performance of Total Hip Replacement (THR) models. The importance of these factors needs to be considered in both experimental setups and computational methods. It is needed to consider which factors are essential to be included and which factors might be neglected in the processes. In order to reduce the expenses of experimental simulations used to validate computational models, the latter should also act as a preexperimental design tool to optimize its configuration, i.e. to find which parameters are more important to replicate and which values of these parameters are more indicative of the behavior of the system. This can be achieved through the implementation of probabilistic analyses together with finite element analysis. In probabilistic studies on THR models, it has been shown that there are several important random variables, such as bone geometry, implant design geometry, cement geometry, material properties, magnitude and direction of applied loads, and bone-implant relative position that can affect performance (Gonzalez 2009, Kharmanda and Antypas 2018). In order to improve the stem design, 
several considerations can be taken into account. The first stage is to consider that the rectangular section stem as more reliable than the circular section stem (Kharmanda et al. 2017). Here, the rectangular section stem allows a secure diaphyseal press-fit in the frontal plane of the femoral canal. This provides excellent rotational stability and increases primary mechanical fixation (Gonzalez 2009), and also reduces wear as mention in Kharmanda and Albashi (2011). The second stage is to consider the shouldered stem as more reliable than the non-shouldered one. The previous numerical results of Kharmanda et al. (2012) have shown that the shouldered stem leads to a good reduction of the maximum stress values in the different bone layers relative to the non-shouldered stem. The third stage concerns the used materials. Here, Kharmanda et al. (2012) concluded that when using titanium alloy $(\mathrm{Ti}-6 \mathrm{Al}-4 \mathrm{~V})$ for both cup and stem, and ceramic alloy $\left(\mathrm{Al}_{2} \mathrm{O}_{3}\right)$ for both liner and ball, it provides minimum stress transfer values to adjacent bone region. This result is shown to be validated by experimental works in literature (see Garino 2000; Knahr and Pospischill 2006). In this paper, shape optimization will be used to produce hollow stems and probabilistic analysis will be applied to study the boundary conditions change.

\section{Material and methods}

\subsection{Mechanical Properties}

It is well known that the bone materials as well as their structure are quite complicated. It is difficult to determine the material properties (Young's modulus, Poisson's ratio, strength properties, shear modulus, viscoelastic properties, etc.). The bone has also to adapt to the actual loading. It is considered to be an anisotropic material and to determine its stiffness, 21 material parameters are needed (Mackerle 2006). In the present analysis for simplicity, the material properties of bone have been assumed as linearly elastic and isotropic (Kharmanda 2015). The mechanical properties of different components are presented in Table 1.

\begin{tabular}{|c|c|c|c|}
\hline Materials properties & Cortical bone & Cancelleous bone & Stem (Ti6Al4V) \\
\hline Young's modulus (MPa) & 16200 & 389 & 100000 \\
\hline Poisson's ratio & 0.36 & 0.3 & 0.32 \\
\hline Density $\left(\mathbf{g r} / \mathbf{c m}^{\mathbf{3}}\right)$ & 1.99 & 0.5 & 4.43 \\
\hline Yield stress $(\mathbf{M P a})$ & 114 & 3.89 & 830 \\
\hline
\end{tabular}

Table 1. Mechanical properties of different components (McNamara et al. 1997)

The success of hip replacement is directly related to the ability of transferring the load uniformly from the replaced components to all surrounding bone. A region of the bone which is unloaded by the presence of the prosthetic components will undergo resorption (Wolf's law (1986)). Bone resorption leads to loosening and eventually loss of functionality of prostheses. For this reason, the minimum stress values in the bone has to be increased, otherwise bone will die around implant due to the lack of use. To get a good fixation, it is the objective of coupling of prosthetic components to the musculoskeletal system so that prosthetic and natural elements may act together in a harmonious manner.

\subsection{Boundary conditions}

Traditionally, by considering the femur in isolation, it has been believed that the effect of the body weight on the projecting neck and head places is the superior aspect of the neck in tension. A minority view has proposed that this region is in compression because of muscular forces pulling the femur into the pelvis. Rudman et al. (2006) hypothesized that under physiological loading the majority of the proximal femur is in compression and that the internal cancellous structure functions as an arch, 
transfers compressive stresses to the femoral shaft. Hence, a better understanding of the stress distribution in the proximal femur may lead to improvements in prosthetic devices and an appreciation of the effects of various surgical procedures that affect load transmission across the hip. The muscles apply forces that pull the femoral head into the acetabulum and should not be ignored. Most studies assume the traditional model and do not include ligaments, muscles or the acetabulum (Kharmanda and Albashi 2011). It is proposed that the horizontal compressive forces required to provide the abutments in the femur are generated on the lateral side by the ligaments and muscles connecting the femur to the pelvis and on the medial aspect by the acetabulum. In addition, Rudman et al. (2006) studied whether reasonable boundary conditions could be found to support this hypothesis. Their results demonstrated that when ligamentous and muscular forces are included, the stresses in proximal femur are predominantly compressive. This would appear to make better use of the mechanical properties of bone, which is stronger in compression than in tension (Keaveny 2001). For example, the Ultimate Tensile Strength equals to $132 \mathrm{MPa}$ while the Compressive Strength equals to $192 \mathrm{MPa}$. It is noted that none of these ligaments restrict flexion. However, all muscle forces could not be included because the ligaments are almost invariably cut during surgery. As a representative daily loading condition, three loading cases of one-legged stance (L1), extreme ranges of motion of abduction (L2), and adduction (L3) are assumed (Beaupré et al., 1990), as shown in Fig. 1.

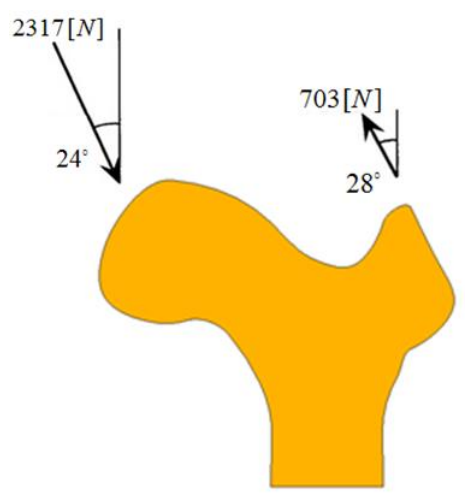

$a$

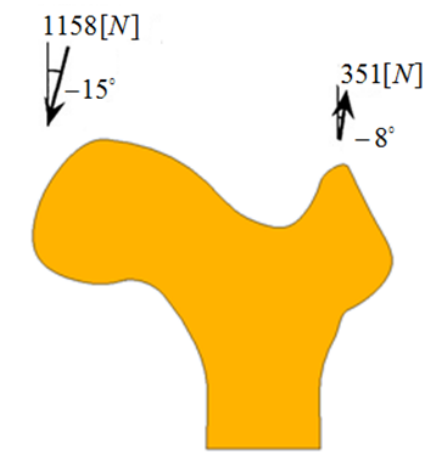

$b$

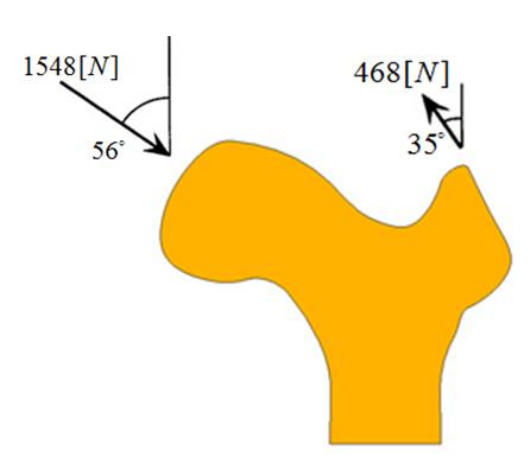

$c$

Fig. 1. a) One-legged stance case (L1), b) Abduction case (L2), and c) Adduction case (L3)

The used components of different forces corresponding to the three loading cases: $L_{1}, L_{2} \& L_{3}$ that are presented in Fig. 1 are shown in Table 2.

\begin{tabular}{|c|c|c|c|c|}
\hline \multirow{2}{*}{$\begin{array}{c}\text { Case } \\
\text { Number }\end{array}$} & \multicolumn{2}{|c|}{ Body Forces } & \multicolumn{2}{c|}{ Muscle Forces } \\
\cline { 2 - 5 } & $F_{x}^{B}[\mathbf{N}]$ & $F_{y}^{B}[\mathbf{N}]$ & $F_{x}^{M}[\mathbf{N}]$ & $F_{y}^{M}[\mathbf{N}]$ \\
\hline Case 1: (L1) & 942,41 & $-2116,68$ & $-330,04$ & 620,71 \\
\hline Case 2: (L2) & $-299,71$ & $-1118,54$ & 48,85 & 347,58 \\
\hline Case 3: (L3) & 1283,35 & $-865,63$ & $-268,43$ & 383,36 \\
\hline
\end{tabular}

Table 2. Force components

The objective of fixation is to eliminate the relative motion between loaded implant and supporting bone, in order to produce an acceptable limit of contact stresses on bone, and to maintain resultant stresses in bone close to the normal physiologic pre-implantation level. For solid stem, ingrown fixation by tissue should be happened by the bone growth into porous portions of coated stem. However, for hollow stem, the bone growth also happens into the holes. Thus, there is a strong need to generate holes which can be carried out using the topology optimization technology at the conceptual design stage (Kharmanda and Albashi 2013). 


\subsection{Shape optimization for hollow stem}

The objective is to minimize the structural volume and the bone stresses subjected to the yield stress values for all layers. Here, a multi-objective optimization problem is created. The structural volume minimization is related with holes that lead to improve the fixation. The second and third objectives seek to obtain a homogeneous stress distribution in both cortical and cancellous layers. This can be called a vector objective function.

$$
\{\mathbf{F}(\mathbf{x})\}=\left\{\begin{array}{l}
f_{1}\left(x_{i}\right) \\
f_{2}\left(x_{i}\right) \\
f_{3}\left(x_{i}\right)
\end{array}\right\}=\left\{\begin{array}{c}
\text { Volume }_{1}\left(x_{i}\right) \\
\sigma_{\text {max }}^{1}\left(x_{i}\right)-\sigma_{\min }^{1} \\
\sigma_{\text {max }}^{2}\left(x_{i}\right)-\sigma_{\min }^{2}
\end{array}\right\}
$$

where $\sigma_{\max }^{1}\left(x_{i}\right)$ and $\sigma_{\min }^{1}\left(x_{i}\right)$ are respectively the maximum and the minimum von-Mises (VM) stresses of the cortical bone. $\sigma_{\max }^{2}\left(x_{i}\right)$ and $\sigma_{\min }^{2}\left(x_{i}\right)$ are respectively the maximum and the minimum von-Mises stresses of the cancellous bone. The used optimization method is zero order problem (curve fitting method or sub-problem) implemented in ANSYS software. However, when using ANSYS, a single objective function can be considered. Therefore, a multiplication form of the objective function is proposed. This way, the shape optimization problem seeks to minimize the structural volume and the bone shielding stress difference, subjected to yield stress values for the maximum stress and the target stress values for the minimum stress values. The mathematical description can be written as follows:

$$
\begin{aligned}
\min & : F\left(x_{i}\right)=\operatorname{Volume}\left(x_{i}\right) \cdot \prod_{l=1}^{L_{n}}\left(\sigma_{\max }^{l}\left(x_{i}\right)-\sigma_{\min }^{l}\left(x_{i}\right)\right) \\
\text { s.t. } & : \sigma_{\max }^{1}\left(x_{i}\right)-\sigma_{y}^{1}\left(x_{i}\right) \leq 0 \\
& : \sigma_{\min }^{2}\left(x_{i}\right)-\sigma_{T}^{2}\left(x_{i}\right) \leq 0 \\
& : \sigma_{\max }^{M}\left(x_{i}\right)-\sigma_{y}^{M}\left(x_{i}\right) \leq 0 \\
& : x_{i}^{U} \leq x_{i} \leq x_{i}^{L}
\end{aligned}
$$

where $F\left(x_{i}\right)$ is a composite objective function and $x_{i}$ are the design variables representing point coordinates and bounded between lower and upper values $\left(x_{i}^{L} \& x_{i}^{U}\right) . \sigma_{\max }^{l}\left(x_{i}\right), \sigma_{T}^{l}\left(x_{i}\right)$ and $\sigma_{y}^{l}\left(x_{i}\right)$ are respectively the maximum von-Mises, target and yield stresses of the three bone layers $(l=1,2)$. $\sigma_{\max }^{M}\left(x_{i}\right)$ is the maximum VM stress in the metallic stem.

\subsection{Probabilistic analysis of osseointegration}

The design of structures and the prediction of their good functioning lead to the verification of a certain number of rules resulting from the knowledge of physical and mechanical experience of designers. These rules show the necessity to limit the loading effects such as stresses and displacements. Each rule represents an elementary event and the occurrence of several events leads to a failure scenario. Then, the objective is to evaluate the failure probability corresponding to the occurrence of critical failure modes. The failure probability is then calculated by:

$$
P_{f}=\operatorname{Pr}\left[G\left(x_{i}, y_{j}\right)\right]=\int_{G\left(x_{i}, y_{j}\right) \leq 0} f_{Y}\left(y_{j}\right) d y_{1} \ldots d y_{n}
$$

where $G\left(x_{i}, y_{j}\right)$ is a limit state function which is defined by the condition of good functioning of the structure (Kharmanda and El-Hami 2016). The limit state $G\left(x_{i}, y_{j}\right)=0$ is located between the failure state $G\left(x_{i}, y_{j}\right)<0$ and the safety state $G\left(x_{i}, y_{j}\right)>0 . f_{Y}\left(y_{j}\right)$ is the density function of the random variable $\mathbf{Y}$. The probability of failure is related with reliability level $R$ by: 


$$
P_{f}=1-R
$$

Several methods can be used to evaluate the failure probability. The most conservative probability method is Monte Carlo simulation (Haldar and Mahadevan 2000), in which the variable parameters (random variables $(\mathrm{RVs})$ ) are sampled at random from their underlying distributions and the probability of failure (POF) evaluated by solving the model repeatedly. The MCS always provides the correct solution if a sufficient number of trials is evaluated, but it is computationally expensive. In order to reduce this expense, and avoid overlapping of samples and/or lack of samples in some regions of the domain, Latin Hypercube Sampling (LHS) may be used (McKay et al.1979). This Monte Carlo based method partitions the sample space into separate intervals with equal probabilities, and one sample is randomly taken from each interval. For implicit models, finite element method has been combined with probabilistic design methods to allow randomness and variability in the system parameters to be taken into account. Several kinds of uncertainties can be distinguished:

- Physical Uncertainty: Material properties, dimensions, loads, \& ground profiles

- Statistical Uncertainty: Due to limited sample sizes, probabilistic model (distribution type and its parameters) is uncertain - lack of information

- Model Uncertainty: Uncertainty of mathematical models and numerical methods due to simplifying assumptions, unknown boundary conditions, and unknown effects of other variables that are not included in the model, etc.

In the next section, a simple 2D implant-bone study of solid and hollow stem designs is presented taking into account statistical uncertainties.

\section{Results:}

An illustration of the hip replacement is shown in Fig. 2 where both a solid stem and a hollow stem are considered Figs. 2b and c. Fig. 2a shows a 3D model of the studied solid stem while a 2D model is considered for simplicity. Figs. $3 b$ \& c show the different studied parts of the solid stem and of the hollow stem. According to Table 1, the cortical (or impact) bone part is assumed to be homogeneous and isotropic material with Young's modulus $E=16.2 \mathrm{GPa}$ and Poisson's ratio $v=0.36$. The cancellous (or spongy) bone layer was also assumed to be a homogeneous and isotropic material with Young's modulus $E=389 \mathrm{MPa}$ and Poisson's ratio $v=0.3$. However, the marrow part was regarded as a cavity, and was neglected in finite element analysis (Tsubota et al. 2002). Titanium-based alloys (Ti6Al4V) have a Young's modulus $E=100 \mathrm{GPa}$ and a Poisson's ratio $v=0.32$.
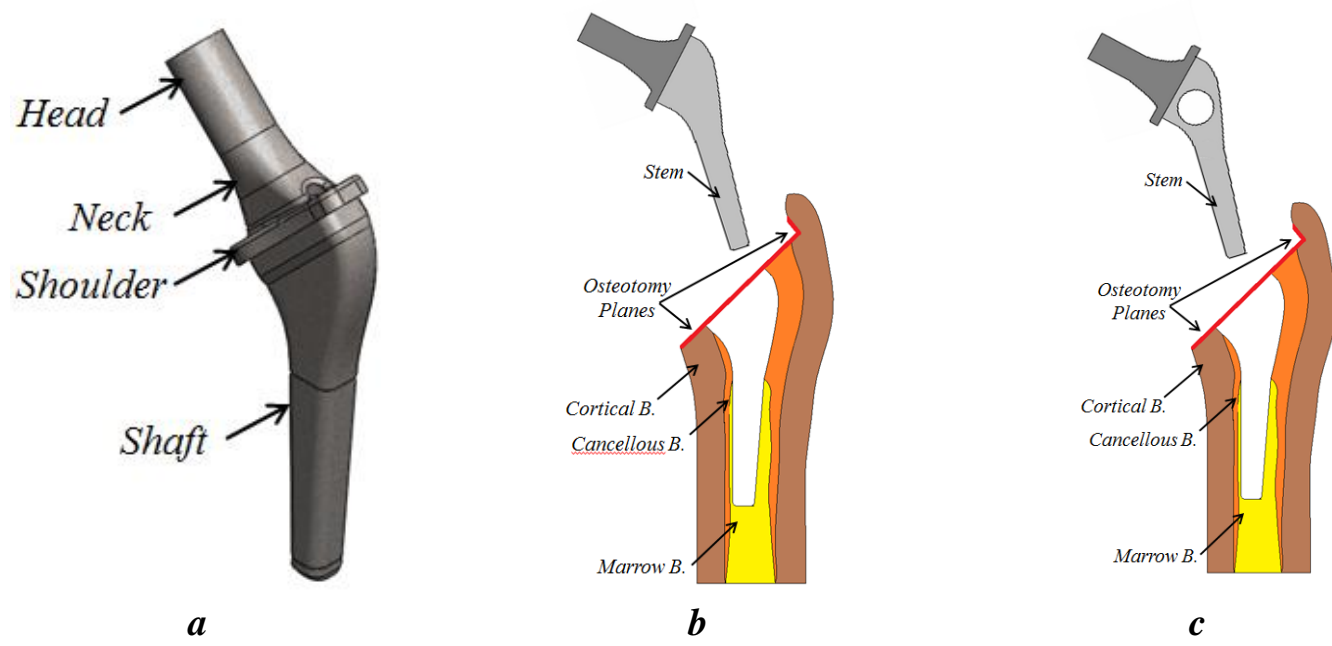

Fig. 2. a) $3 D$ studied stem model, b) Solid stem parts and c) Hollow stem parts 


\subsection{Shape optimization results}

Fig. 3a shows a simplified 2D geometrical model for the studied solid stem with cortical and cancellous bone layers. Fig. $3 \mathrm{~b}$ shows the boundary conditions and the meshing model for the first and third loading cases (L1 and L3) while Fig. 3c shows the boundary conditions and the meshing model for the second loading case L2.
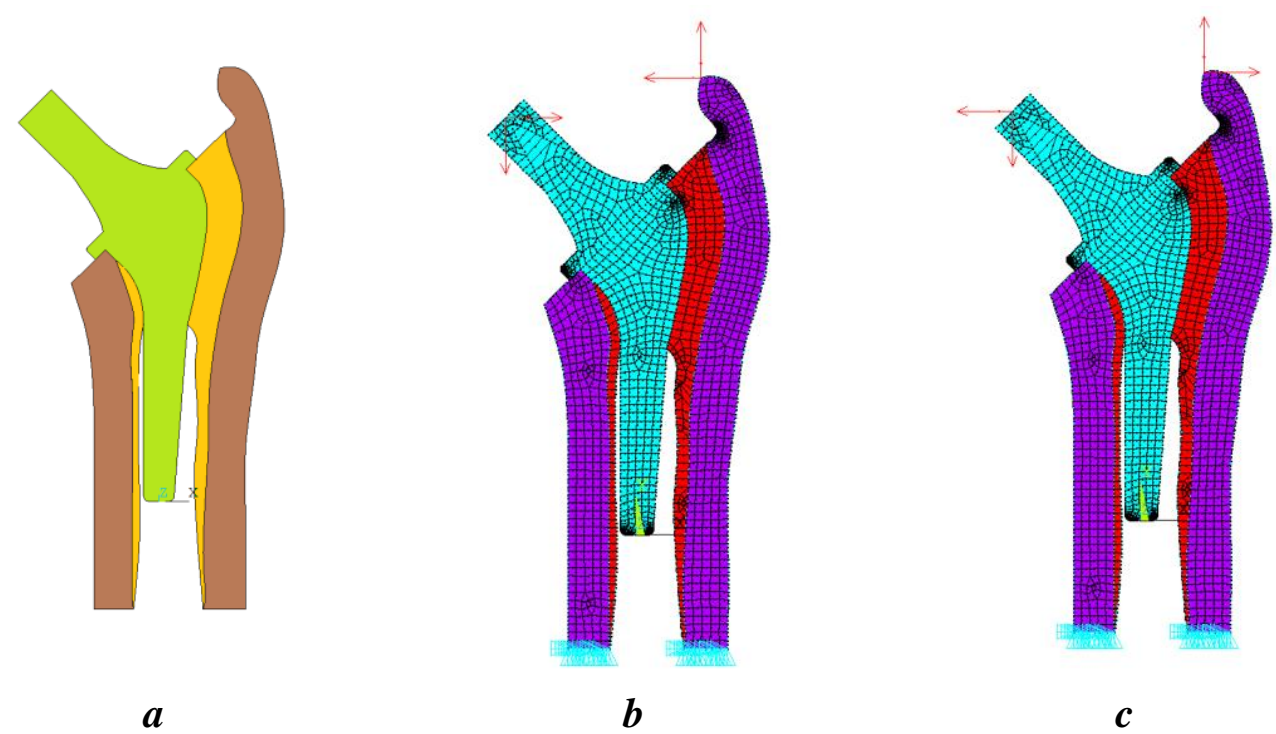

Fig 3. a) Geometry model, b) Boundary conditions for $L 1$ and $L 3$, and $c$ ) Boundary conditions for $L 2$ when considering the solid stem

Table 3 shows the resulting stress VM distribution for the studied solid stem for different loading cases (L1, L2 and L3).

\begin{tabular}{|c|c|c|c|}
\hline \multirow{2}{*}{$\begin{array}{c}\text { Output } \\
\text { Parameters }\end{array}$} & \multicolumn{3}{|c|}{ Solid Stem } \\
\cline { 2 - 4 } & L1 & L2 & L3 \\
\hline$\sigma_{\max }^{1}(\mathrm{MPa})$ & 14.18 & 9.05 & 16.76 \\
\hline$\sigma_{\max }^{2}(\mathrm{MPa})$ & 3.94 & 4.06 & 6.08 \\
\hline$\sigma_{\max }^{M}(\mathrm{MPa})$ & 16.72 & 13.10 & 11.26 \\
\hline Volume $\left(\mathrm{mm}^{3}\right)$ & \multicolumn{3}{|c}{5675.22} \\
\hline
\end{tabular}

Table 3. Numerical simulation results of solid stem for different loading cases

Figs 4a, b and c show the VM stress distribution of different layers for three loading case L1, L2 and L3 respectively when considering the solid stem. This way the porous coated surface between the metallic stem and the bone layers is the single way for fixation. 

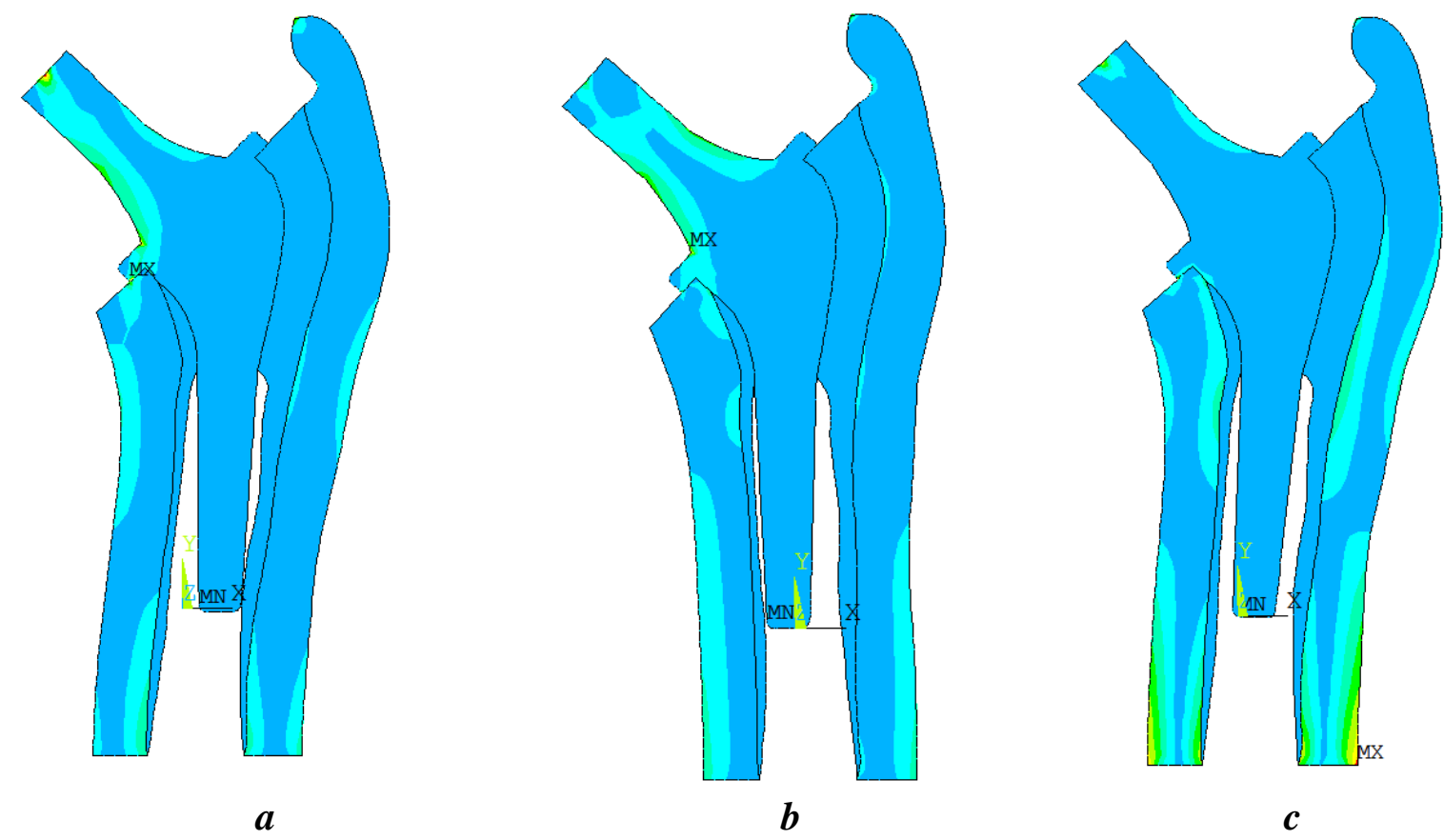

Fig 4. VM stress distribution of different layers a), b), c) for three loading case $L 1, L 2$ and $L 3$, respectively when considering the solid stem

According to these results, the maximum VM stress of cancellous bone has exceeded the yield stress. The change of the boundary conditions may improve the performance. Thus, there is a strong need to introduce gaps or holes in order to allow the cancellous bone to grow into the gaps (Osseosynthesis). Fig. 5a shows a simplified 2D geometrical model for the optimized hollow stem with cortical and cancellous bone layers. Fig. 5b shows the boundary conditions and the meshing model for the first and third loading cases (L1 and L3) while Fig. 5c shows the boundary conditions and the meshing model for the second loading case L2 for the optimized hollow stem.

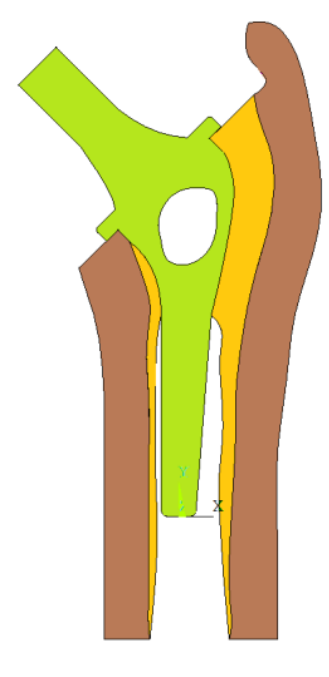

$a$

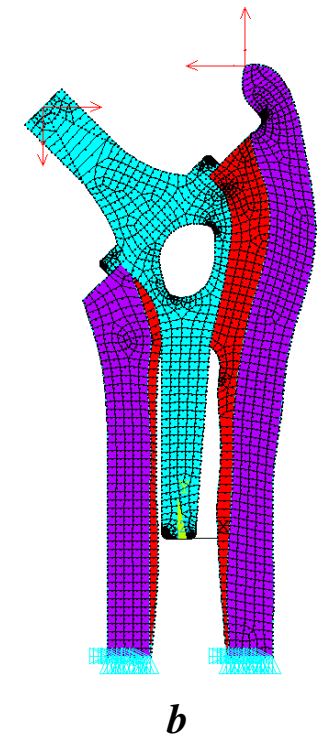

$b$

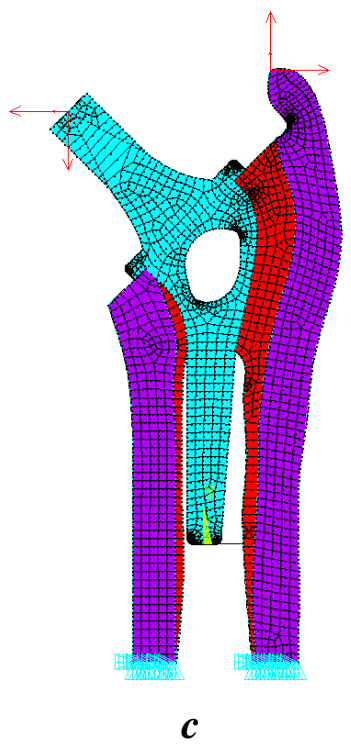

$c$

Fig 5. a) Geometry model, b) Boundary conditions for $L 1$ and $L 3$, and C) Boundary conditions for $L 2$ when considering the hollow stem

Table 4 shows the resulting VM stress distribution for the optimized hollow stem for different loading cases (L1, L2 and L3). The maximum VM stress values are almost close to those of solid stem. 


\begin{tabular}{|c|c|c|c|}
\hline \multirow{2}{*}{$\begin{array}{c}\text { Output } \\
\text { Parameters }\end{array}$} & L1 & L2 & L3 \\
\cline { 2 - 4 } & 13.51 & 9.07 & 16.94 \\
\hline$\sigma_{\max }^{1}(\mathbf{M P a})$ & 3.95 & 3.94 & 6.13 \\
\hline$\sigma_{\max }^{2}(\mathbf{M P a})$ & 17.41 & 12.89 & 14.15 \\
\hline$\sigma_{\max }^{M}(\mathbf{M P a})$ & \multicolumn{3}{|c}{} \\
\hline Volume $\left(\mathbf{m m}^{\mathbf{3}}\right)$ & 5457.40 \\
\hline
\end{tabular}

Table 4. Numerical simulation results of hollow stem for different loading cases

Figs. 6a, $\mathrm{b}$ and $\mathrm{c}$ show the VM stress distribution of different layers for three loading case L1, L2 and L3, respectively when considering the hollow stem. This way the porous coated surface between the metallic stem and the bone layers is the single way for fixation.

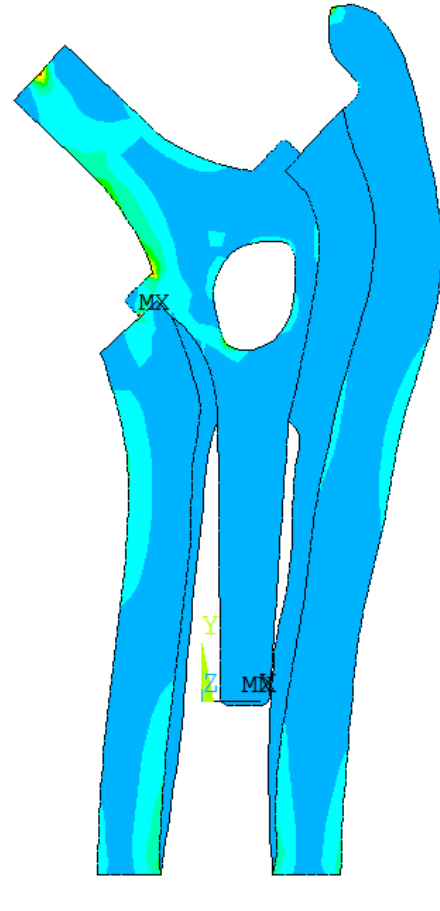

$a$

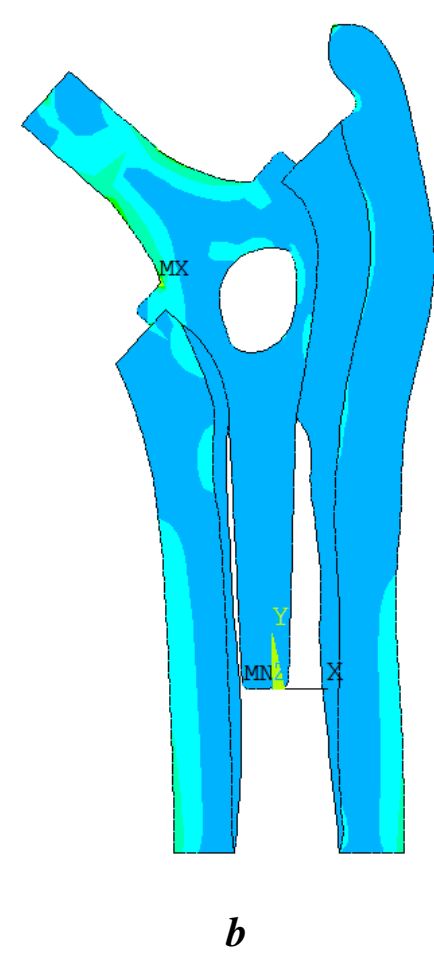

$\boldsymbol{b}$

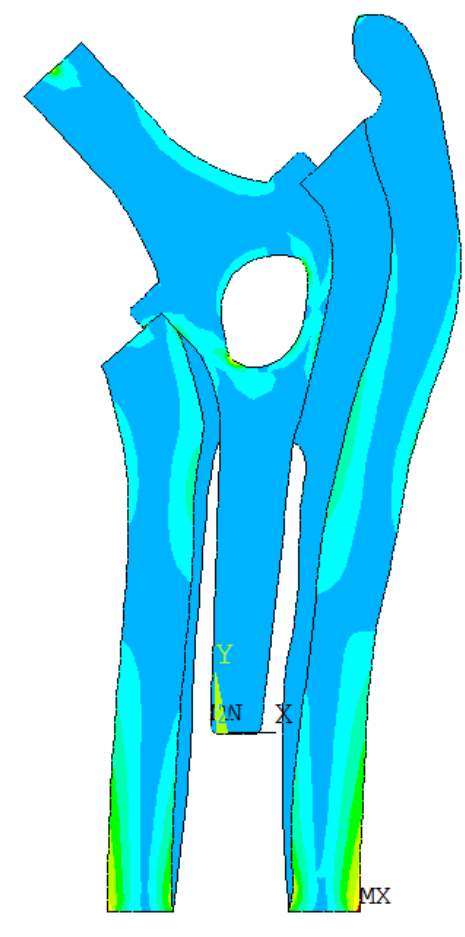

c

Fig 6. VM stress distribution of different layers a), b), c) for three loading case L1, L2 and $L 3$, respectively when considering the hollow stem

The optimized hollow stem model led to a reduction of structural volume by $3.8 \%$. Also, it does not lead to higher stress values relative to the solid stem as shows in Table 4. The optimization process improves the stem performance especially for the ingrowth of bone into holes after a healing period (almost 6 months). When considering the ingrowths of bone, different boundary conditions are considered. This way, the new change in the boundary conditions needs to be determined. Here, the hole is assumed to be filled with the cancellous bone and modeled as shown in Fig. 7. To attend the yield stress, the maximum displacements are: $\delta_{\max }^{x} \approx 0.7, \delta_{\max }^{x} \approx 0.8 \mathrm{~mm}$. 


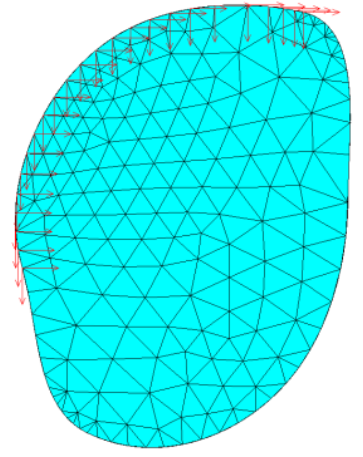

$a$

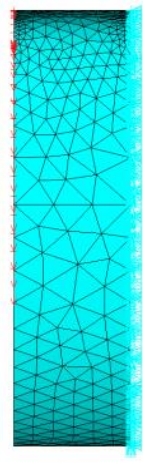

$b$

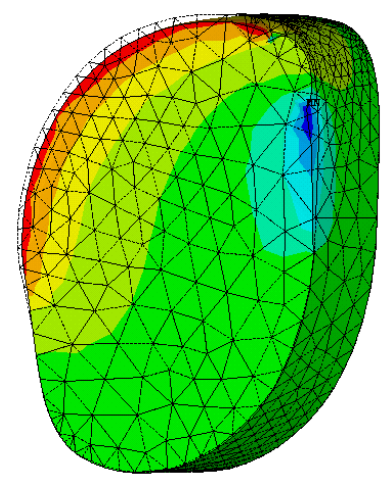

c

Fig 7. a), b) Front and Right views, respectively of the meshing and boundary conditions, and c) Resulting von-Mises displacement distribution for the hole with cancellous bone

Fig. 8a shows a simplified 2D geometrical model for the optimized hollow stem with cortical and cancellous bone layers. Fig. 5b shows the boundary conditions and the meshing model for the first and third loading cases (L1 and L3) while Fig. 3c shows the boundary conditions and the meshing model for the second loading case L2.

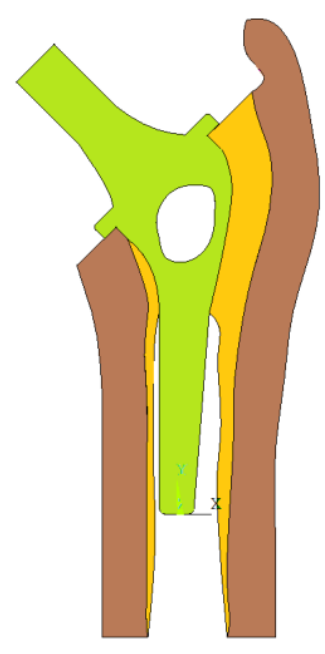

$a$

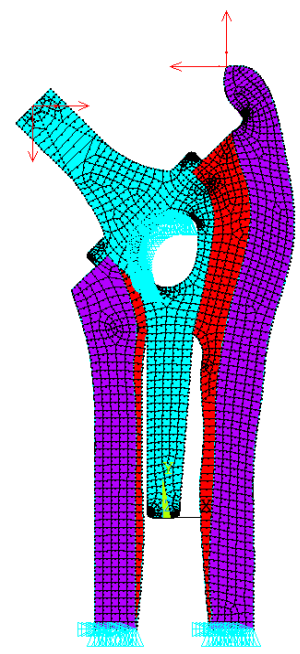

$b$

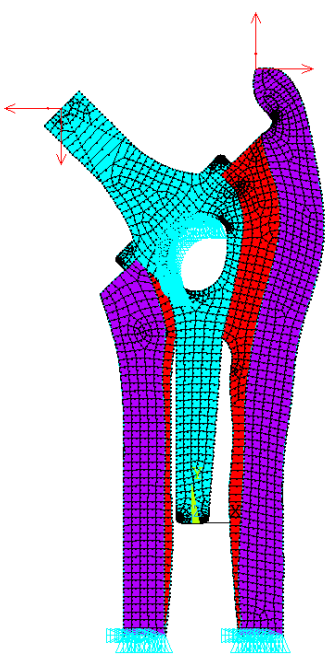

$\boldsymbol{C}$

Fig 8. a) Geometry model, b) Boundary conditions for $L 1$ and $L 3$, and c) Boundary conditions for $\mathrm{L} 2$, when considering the hollow stem with new boundary conditions

Table 5 shows the resulting VM stress distribution for the optimized hollow stem when considering the new boundary conditions for different loading cases (L1, L2 and L3). The maximum stress values are smaller than those of solid stem. 


\begin{tabular}{|c|c|c|c|}
\hline \multirow{2}{*}{$\begin{array}{c}\text { Output } \\
\text { Parameters }\end{array}$} & \multicolumn{3}{|c|}{ Hollow Stem } \\
\cline { 2 - 4 } & L1 & L2 & L3 \\
\hline$\sigma_{\max }^{1}(\mathbf{M P a})$ & 13.04 & 9.07 & 8.27 \\
\hline$\sigma_{\max }^{2}(\mathbf{M P a})$ & 2.07 & 2.05 & 2.46 \\
\hline$\sigma_{\max }^{M}(\mathbf{M P a})$ & 17.16 & 13.79 & 11.51 \\
\hline Volume $\left(\mathbf{m m}^{3}\right)$ & & 5457.40 \\
\hline
\end{tabular}

Table 5. Numerical simulation results of hollow stem for different loading cases after healing

Figs. 9a, b and c show the VM stress distribution of the deformed shape comparing with that of undeformed one for bone layers for the three loading cases L1, L2 and L3 respectively.

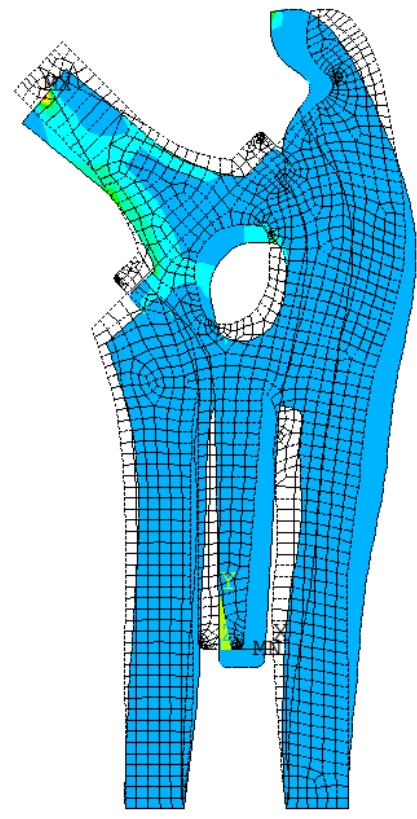

$a$

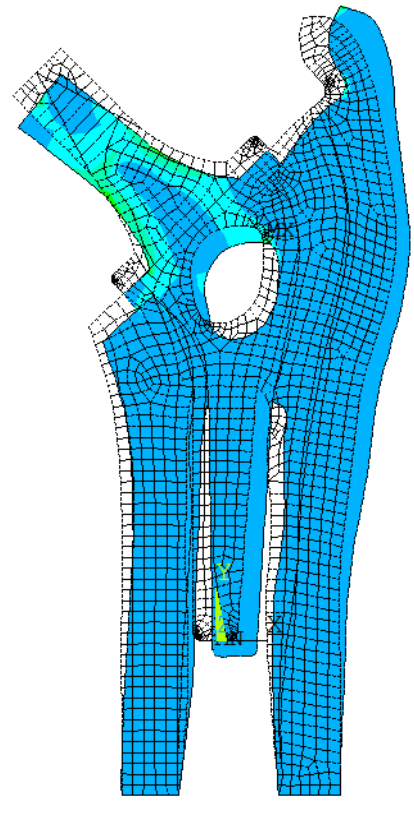

$\boldsymbol{b}$

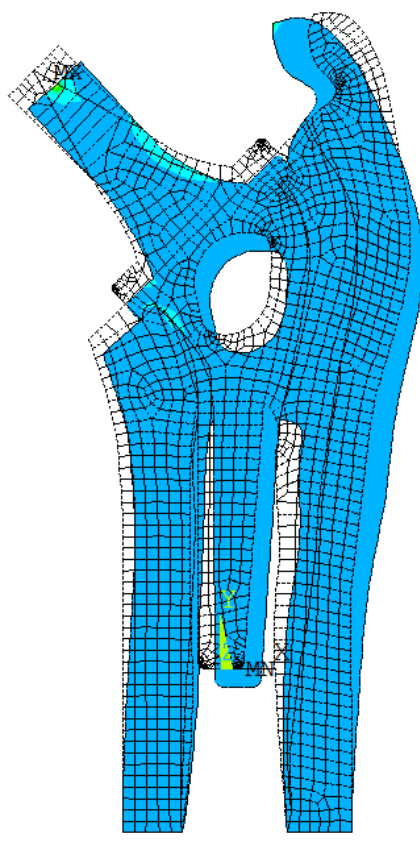

c

Fig 9. VM stress distribution for different layers a), b), c) for the three loading cases $L 1, L 2$ and $L 3$ respectively when considering the optimized hollow stem

Here, we can find the importance of the ingrowth of bone that leads to a good reduction of the maximum stress values for the bone layers. However, these new boundary conditions are uncertain, therefore, there is a strong need to consider the probabilistic analysis of this case.

\subsection{Probabilistic analysis results}

The ANSYS Probabilistic Design System (PDS) analyzes a component or a system involving uncertain input parameters. These input parameters (geometry, material properties, boundary conditions, etc.) are defined in the ANSYS software. The variation of these input parameters is defined as random input variables and is characterized by their distribution type (Gaussian, lognormal, etc.) as well as their distribution parameters (mean values, standard deviation, etc.). Any interdependencies between random input variables are also defined as correlation coefficients. The important results are defined as random output parameters. During a probabilistic analysis, ANSYS executes multiple analysis loops to compute the random output parameters as a function of the set of random input variables. The values for the input variables are generated either randomly (using Monte Carlo simulation) or as prescribed samples (using Response Surface Methods). 
The deterministic model has two parameters that are regarded as random input variables. Thus, two sources of uncertainty, or random variables, were considered in the present investigation: the magnitude of horizontal and vertical displacements. Both parameters have been demonstrated to influence bone performance in current probabilistic and parametric study. The means for these parameters are: $\delta_{\max }^{x} \approx 0.7, \delta_{\max }^{y} \approx 0.8 \mathrm{~mm}$. And the standard deviations are considered proportional of the mean values by $10 \%$ with a normal distribution law. The selection of the output or failure criterion plays an important role in the results. The maximum stress of cancellous bone $\left(\sigma_{\max }^{2}\right)$ can be considered as a limit state or the most importance output parameter. To assess the accuracy of the results, the convergence of the mean value of the output parameters was plotted, with a high confidence interval of 99.9\% (reliability level). The probability of failure is noted to be $0.1 \%$. Using Sampling Method, Monte Carlo based simulations were run, with 30000 simulations for each loading case (L1, L2 and L3). A sensitivity analysis was performed to assess the influence of each parameter on the bone stresses. The evaluation of the sensitivities was based on both Rank-order correlation coefficients between the input parameters and the output parameters. Table 6 shows the statistical results of the random input variables.

\begin{tabular}{|c|c|c|c|c|c|c|}
\hline \multirow{2}{*}{$\begin{array}{c}\text { Input } \\
\text { Parameters }\end{array}$} & \multicolumn{3}{|c|}{ Horizontal Displacement $\delta_{\max }^{x}[\mathrm{~mm}]$} & \multicolumn{3}{|c|}{ Vertical Displacement $\delta_{\max }^{y}[\mathrm{~mm}]$} \\
\hline & L1 & $\mathbf{L} 2$ & $\mathbf{L 3}$ & L1 & $\mathbf{L} 2$ & $\mathbf{L 3}$ \\
\hline Mean & \multicolumn{3}{|c|}{0.7} & \multicolumn{3}{|c|}{0.8} \\
\hline $\begin{array}{l}\text { Standard } \\
\text { Deviation }\end{array}$ & \multicolumn{3}{|c|}{0.07} & \multicolumn{3}{|c|}{0.08} \\
\hline Minimum & 0.4 & 0.39 & 0.42 & 0.47 & 0.46 & 0.45 \\
\hline Maximum & 0.988 & 0.987 & 0.979 & 1.17 & 1.12 & 1.14 \\
\hline
\end{tabular}

Table 6. Statistics of the random input variables

Table 7 shows the statistical results of the random output parameter $\sigma_{\max }^{2}$ (the limit state function).

\begin{tabular}{|c|c|c|c|}
\hline & L1 & L2 & L3 \\
\hline Mean & 2.079 & 2.048 & 2.462 \\
\hline Standard Deviation & 0.2130 & 0.2283 & 0.2421 \\
\hline Minimum & 1.647 & 1.131 & 1.530 \\
\hline Maximum & 3.144 & 2.988 & 3.482 \\
\hline
\end{tabular}

Table 7. Statistics of the random output parameter

The sample history values are a function of the number of simulation loops. The plot is only applicable for Monte Carlo Simulation results. For the mean and standard deviation, the curves indicate that the "true" mean value and standard deviation are in fact between the upper and lower confidence bounds with $99.9 \%$. For the minimum value, only the upper confidence bound is shown. The curve means that the "true" minimum value is below that curve with $99.9 \%$ sureness. The same applies analogously for the maximum value, where only the lower confidence curve is shown. Fig. 10 shows that the convergence of the mean value of the critical maximum VM stress of cancellous bone improved gradually as a function of the number of simulations, it is also shows that it stabilized after more than 20000 simulations. 


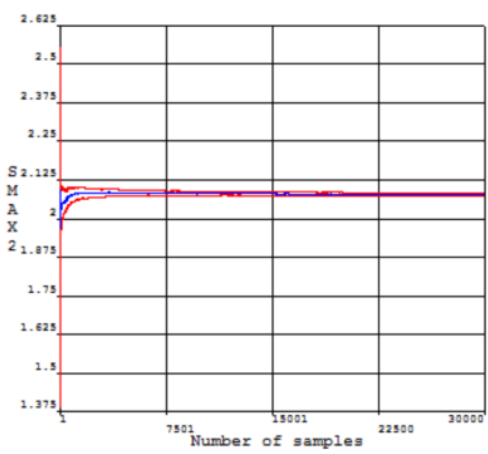

$a$

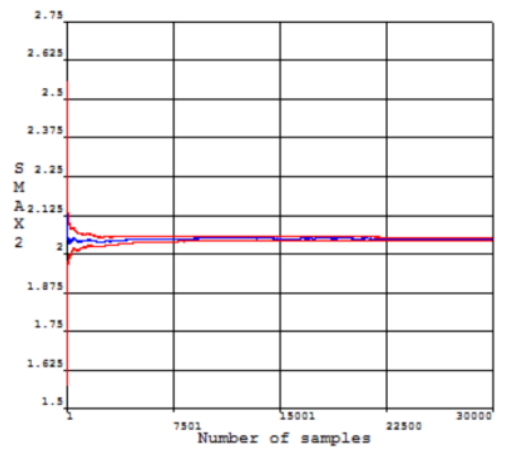

$b$

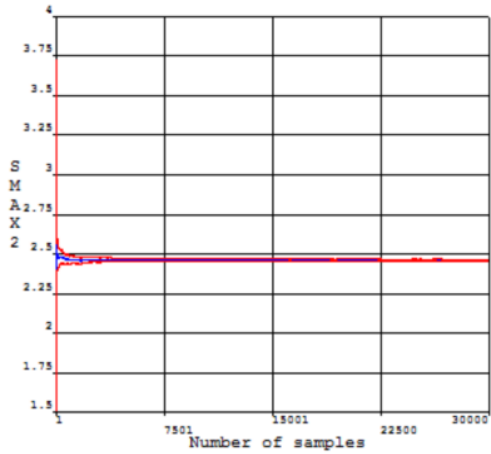

c

Fig. 10. Mean value history of the output parameter for the three loading cases a) $L 1, b) L 2$, and c) $L 3$

Figs. 11 and 12 show histograms of input parameters $\delta_{\max }^{x}$ and $\delta_{\max }^{y}$, using normal distribution law.
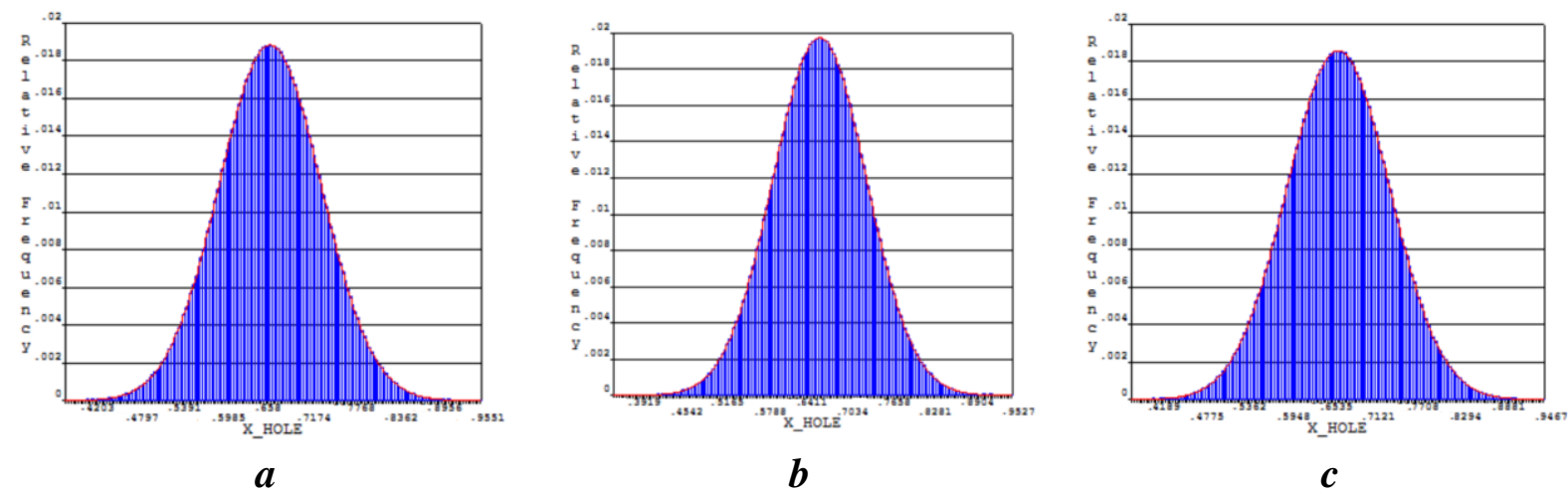

Fig. 11. Histogram of the input parameters $\delta_{\max }^{x}$ for the three loading cases a) $\left.L 1, b\right) L 2$, and c) $L 3$
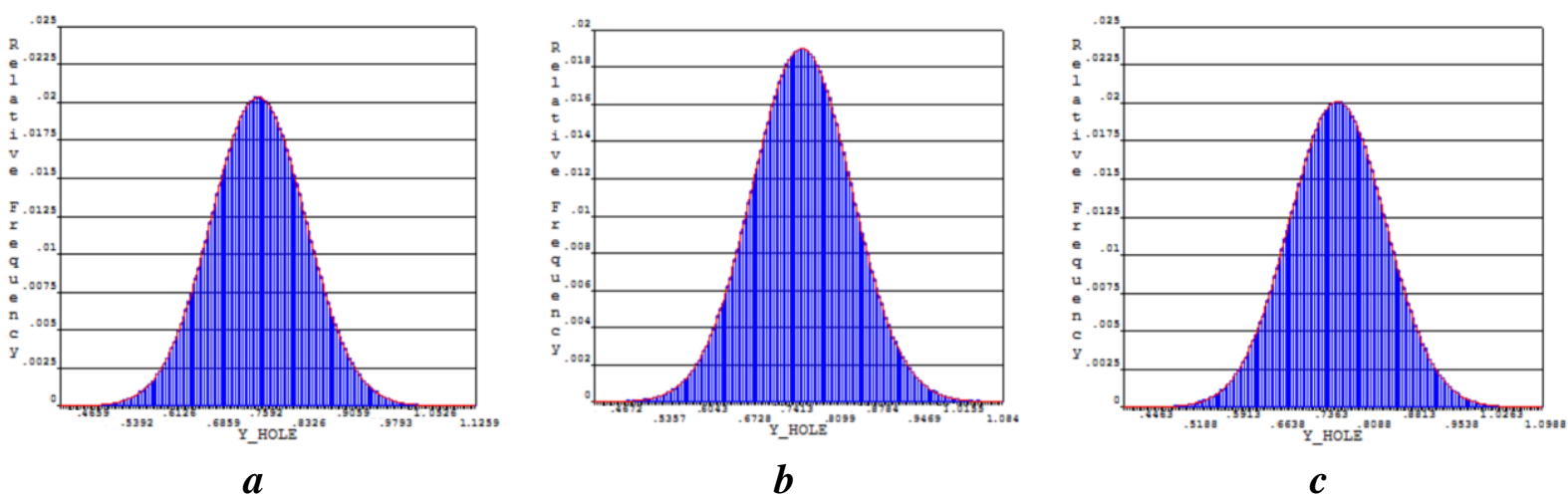

Fig. 12. Histogram of the input parameters $\delta_{\max }^{y}$ for the three loading cases a) $\left.L 1, b\right) L 2$, and c) $L 3$

Fig. 13 shows the histogram of the output parameter $\sigma_{\max }^{2}$ for three loading cases a) L1, b) L2, and c) L3. 


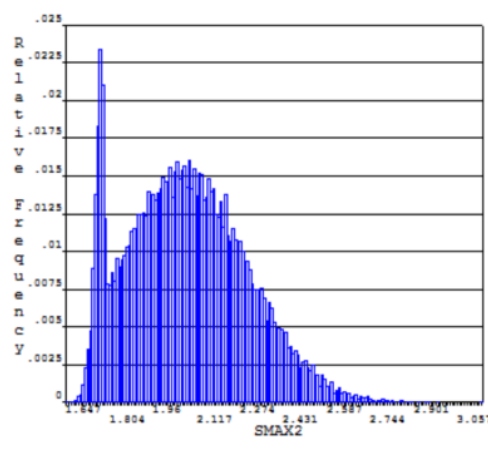

$\boldsymbol{a}$

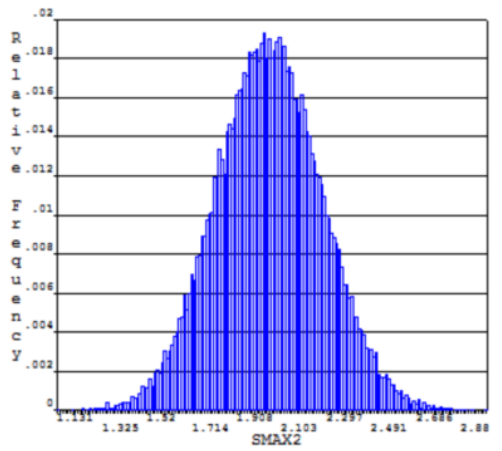

$\boldsymbol{b}$

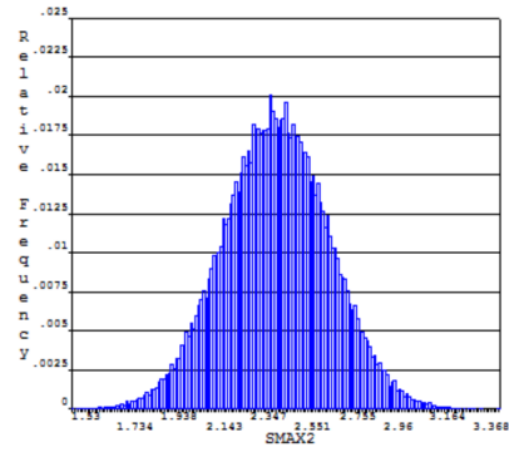

c

Fig. 13. Histogram of the output parameter $\sigma_{\max }^{2}$ for the three loading cases a) $\left.L 1, b\right) L 2$, and c) $L 3$

ANSYS PDS calculates an appropriate number of classes based on the number of samples. The number of classes is equal to the number of bars shown in the histogram. The range between the smallest and largest sample value has been divided into classes of equal width. A histogram is derived by counting the number of hits in the individual classes and dividing this number by the total number of samples. Hence, a histogram represents the relative frequencies of the random quantity that is plotted for. Next, the cumulative distribution function (CDF) is plotted with either a total number of 100 points or using the sample size as the number of points, whichever is lower. If the sample size is less than 100, all samples are represented in the plot. If the sample size is larger than 100, the probabilistic design tool classifies the sample into 100 classes of appropriate size. For Monte Carlo simulation methods, a confidence level of $99.9 \%$ is used to plot confidence bounds around the cumulative distribution function. Fig. 14 shows the change of probability of failure in percentile relative to the studied output parameter (or limit state). The minimum value of stress has found to meet the highest reliability level or the lowest probability of failure $\left(P_{f}=0.1 \%\right)$.
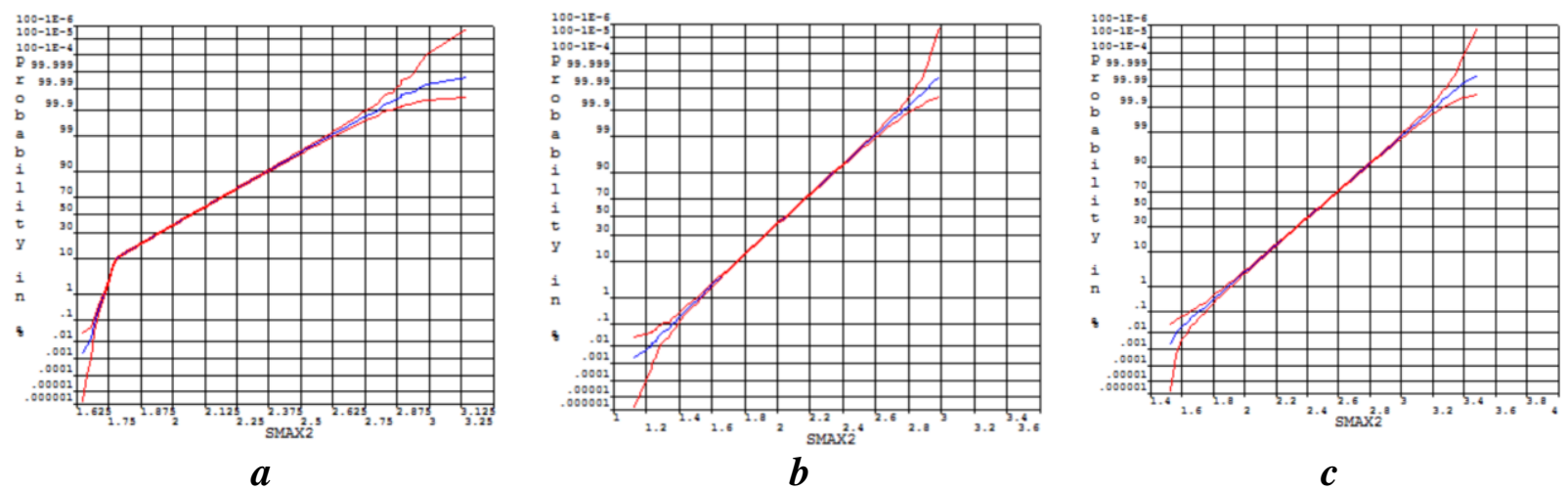

Fig. 14. Probability of the output parameter for the three loading cases a) $L 1, b) L 2$, and c) $L 3$

The evaluation of the probabilistic sensitivities is based on the correlation coefficients between all random input variables and a particular random output parameter. Either Spearman rank order correlation coefficients or Pearson linear correlation coefficients are used. To plot the sensitivities of a certain random output parameter, the random input variables are separated into two groups: those that are significant (important) and those that are insignificant (not important) for the random output parameter. Only the significant random input variables are included in the sensitivity plots. Fig. 15 shows the sensitivity measurements of the output parameter with respect to the input random variables. Here, we note that the vertical displacement influence is much bigger than horizontal one. 


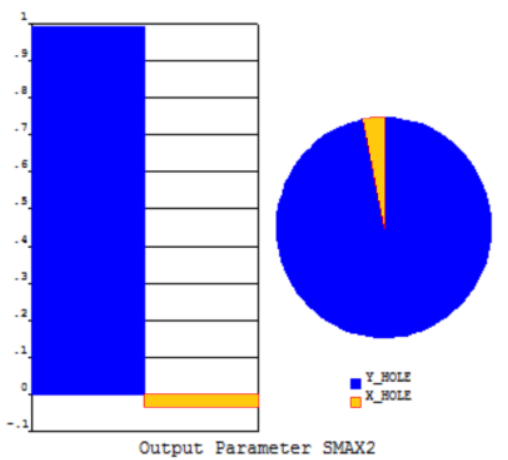

$a$

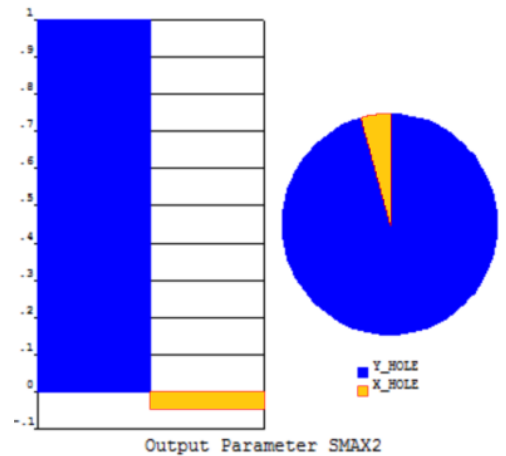

$\boldsymbol{b}$

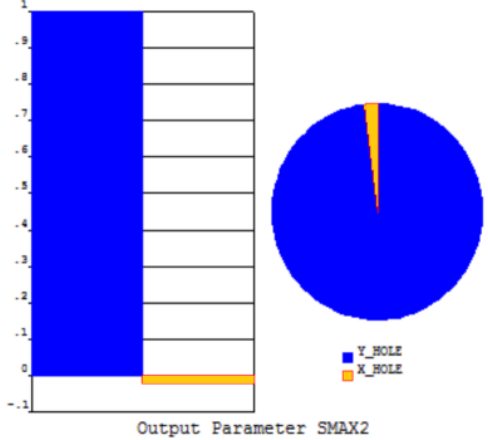

$c$

Fig. 15. Sensitivity of the output parameter for the three loading cases a) $L 1, b) L 2$, and c) $L 3$

\section{Discussion}

The shape optimization is integrated into the stem design to provide with smooth inner hole boundaries. The holes can simply be considered as circles. This way the design variables can be presented by the diameter and the circle center coordinates. Then, three design variables are considered. However, the studied problem is generalized to model the hole by splines. In this problem, four points are varied for the hole that led to consider eight design variables for the studied hole. For the same boundary conditions, a big difference for the different stress values is not noted. Using the hollow stem, a much more variable model than the solid one is got that allows controlling the stress shielding distribution. The main importance of this work is to study the probability of the cancellous bone that grows in the hole. This way a significant change of boundary conditions will happen that may lead to reduce the deformation in the structure. This role becomes very significant after the bone ingrowth (healing period). A probabilistic analysis is carried out to identify the probable change of the hole boundary conditions. Monte Carlo method is used as a robust tool with a big number of simulations to provide with accurate results. The mean value of the critical maximum VM stress of cancellous bone converged after more than 20000 simulations. The sensitivity of the output parameter with respect to the input random variables is finally carried out in order to determine the input parameter influence. It is noted that the vertical displacement influence is always much bigger than the horizontal one for the three studied loading cases.

\section{Conclusions}

The integration of holes into stem design leads to more reliable fixation relative to the solid stems with porous coated surfaces. Furthermore, it allows us to parameterize the inner geometry of the studied stem while the solid stem possesses the outer geometry parameterization possibility. These advantages also allow us to control the stress shielding distribution adjacent to bone layers. The numerical validation using shape optimization technology shows the importance of optimum hole distribution into stem design. A detailed numerical application on different loading cases reported in the literature show the importance of the proposed strategy relative to existing ones. This result allows us at least to parameterize and optimize the inner stem geometry that leads to a control of the stress distributions relative to solid stem. The importance is to largely reduce the bone stresses after healing period. A probabilistic analysis is carried out to find the different probabilistic bounds with a high reliability levels. Here, the variation interval of the cancellous bone stresses are found. These results show that the hollow stem is much more advantageous than the solid stem especially for young patients that leads to a good fixation possibility. As a future work, a mathematical model of the bone ingrowths behavior can be defined considering several numerical trials and clinical observations. 


\section{References}

Beaupré, G.S.; Orr, T.E.; Carter, D.R. (1990): An approach for time-dependent bone modelingand remodelingapplication: a preliminary remodeling simulation. Journal of Orthopaedic Research 8 (5), 662-670.

Garino, J-P. (2000): Modern ceramic-on-ceramic total hip systems in United State, Clinical Orthopaedics 2000; Oct., (379); pp 41.

Gonzalez, C.D. (2009): Probabilistic Finite Element Analysis of Un-cemented Total Hip Replacement, PhD thesis, School of Engineering Sciences, Bioengineering Sciences Research Group, University of Southampton, March 2009

Haldar, A.; Mahadevan, S. (2000): Probability, reliability and statistical methods in engineering design. New York, USA: John Willey \& Sons; 2000.

Keaveny, T.M. (2001): Strength of Trabecular Bone. In Bone Mechanics Handbook. Volume 16. Second edition. Edited by Cowin SC. London, CRC Press; 2001::16.1-16.42.

Kharmanda, G.; Albashi, L. (2011): Numerical Study for Increasing Efficiency of Artificially Un-cemented Hip Joint, Research Journal of Aleppo University, Engineering Science Series, vol. 101, 2011.

Kharmanda, G.; Mulki, S.; Sabsabi, Y. (2012): Compter-Aided Design of Internal Replacement Models In Orthopedics Surgery, Research Journal of Aleppo University, series of Engineering Sciences, vol. 105, 2012

Kharmanda, G.; Albashi, L. (2013): Integration of Topology Optimization into Osteo-Replaced Joint Design, Research Journal of Aleppo University, series of Engineering Sciences, vol. 113, 2013

Kharmanda, G. (2015), Reliability analysis for cementless hip prosthesis using a new optimized formulation of yield stress against elasticity modulus relationship, Materials and Design (January 2015), 65: 496-504

Kharmanda, G.; El-Hami, A. (2016): Reliability in Biomechanics, ISTE \& Wiley, ISBN: 9781786300249, pp 266, November 2016

Kharmanda, G.; El-Hami A.; Ibrahim MH (2017), Integration of reliability and optimization concepts into orthopedic prosthesis design: Application on hip prosthesis design, Journal of Uncertainties and Reliability of Multiphysical Systems, 17-1, Jan 2017

Kharmanda G.; Antypas I. (2018) Reliability-based design algorithm for artificially replaced hip prosthesis considering material property uncertainty, Journal of Advances in Engineering Research, AER, 2018 Advances in Engineering Research (AER), vol 157, pp 44-50

Knahr, K.; Pospischill, M. (2006): Revision Strategies in Total Hip Arthroplasty with Regard to Ceramic Articulations, European Musculoskeletal Review, 2006, pp 54-55.

McKay, M.D.; Beckman, R.J.; Conover, W.J. (1979): A comparison of three methods for selecting values of input variables in the analysis of output from a computer code. Technometrics 1979; 42:55-61.

McNamara, B.P.; Cristofolini, L.; Toni, A., Taylor, D. (1997): Relationship between bone-prosthesis bonding and load transfer in total hip reconstruction, J. Biomech. 30, (1997) 621.

Mackerle, J. (2006): Finite element modeling and simulations in orthopedics: a bibliography 1998-2005, J. Computer Methods in Biomechanics and Biomedical Engineering, Vol. 9, No. 3, June 2006, 149-199

Rudman, K.E.; Aspden, R.M.; Meakin, J.R., (2006): Compression or tension? The stress distribution in the proximal femur, BIOMEDICAL ENGINEERING ONLINE 5: Art. No. 12 FEB 202006

Tsubota, K.; Adachi, T.; Tomita, Y. (2002): Functional adaptation of cancellous bone in human proximal femur predicted by trabecular surface remodelingsimulation toward uniform stress state, Journal of Biomechanics 35 (2002) 15411551

Wolff, J. (1986): The Law of Bone Remodeling, Berlin Heidelberg New York: Springer, 1986 (translation of the German 1892 edition) 